\title{
Altruism and Career Concerns
}

\author{
OLEG SHCHETININ
}




\title{
Altruism and Career Concerns *
}

\author{
Oleg Shchetinin \\ Toulouse School of Economics
}

September 2009

\begin{abstract}
The paper studies the impact of altruism on Agent's motivation in the career concerns model. The paper shows the new channel of interaction between intrinsic and extrinsic motivation. The common point in the literature is that intrinsic motivation can be crowded out by the extrinsic incentives. My paper shows that crowding effect can go in the opposite direction: extrinsic incentives can be lessened for the intrinsically motivated agent. The analysis shows that altruism can decrease effort, though conventional wisdom suggests that effort should always be higher for the more altruistic worker. The key for the result is the distinction between current and anticipated altruism. The paper also studies the effect of altruism on wage.

The model has a number of other interesting features. It gives an example of winner's blessing, it shows that ambitions can hinder altruistic relation. The model can be naturally applied to the workplace relation; another application is the local public good provision.
\end{abstract}

Keywords: Extrinsic and intrinsic motivation; Career concerns; Altruism; Behavioral Economics.

JEL Classification Numbers: M52, D82, D64.

\section{Introduction}

Most of the contract and incentives theory literature is based on the selfishness assumption - see Bolton and Dewatripont (2005), Laffont and Martimort (2002). While economists typically posit self-interest, there is strong evidence from the field and laboratory, suggesting that economic agents internalize the welfare of the others. For instance, Andreoni (2006) provides a comprehensive survey of evidence for philanthropy and public goods contribution. Rotemberg (2006) surveys the evidence from the field. The prominent evidence for pure altruism going from the lab is the Dictator Game introduced in Kahneman et al. (1986). Overall, according to the experimental studies, some $50 \%$ of people demonstrate altruism in their behavior - see Fehr and Schmidt (2003).

\footnotetext{
*I'm grateful to Jean Tirole for support and encouragement. I'm also grateful to the participants of the Behavioral and Experimental Economics workshop in Toulouse and to the participants of the ASSET-2008 Conference for comments and suggestions.
} 
The fact that altruism of workers may be exploited to improve firm's performance is known to practitioners, in particular to the Human Resources Managers. The popular point is that "business should use human nature to motivate employee. Reciprocal altruism is part of our nature. Businesses should embrace that and use it to their advantage by structuring a work environment that encourages such behavior" " ${ }^{1}$. Clearly, altruism is an important intrinsic motivator for the workers. This motivates the study of the variants of the agency models, which include the altruistic component in preferences.

My paper studies the impact of altruism on the Principal-Agent relation in the career concern situation, in particular the interaction between extrinsic and intrinsic motivation.

The model of the paper demonstrates the new channel of interaction between intrinsic and extrinsic incentives. The literature typically stresses one channel - the intrinsic motivation can be crowded out by the extrinsic incentives - see, e.g. Fehr and Rockenbach (2003). My paper shows that crowding effect can work in the opposite direction - an extrinsic incentive (career concerns) can be lessened for the intrinsically motivated (altruistic) agent.

The evident consequence of the Agent's altruism is partial internalization of the Principal's utility, which weakens moral hazard. It is also clear that, due to this internalization, social welfare should be improved if altruistic relation is established. However, the more detailed analysis uncovers more subtle features of the impact of altruism.

The paper demonstrates that in the dynamic context it is important to distinguish between the two types of altruism - current and anticipated, which affect effort in the opposite directions. The former stimulates the Agent; this results from the internalization of the Principal's benefits, related to output. The latter, on the contrary, lessens career concerns and weakens incentives. Which type of altruism dominates depends on the parameters of the model relative uncertainty about Agent's skill and relative importance of the future. The intensity of altruism magnifies the prevalence of one of the altruism types. In particular, if the anticipated altruism dominates, higher altruism makes its dominance stronger which means that effort decreases with altruism.

The paper also demonstrates that altruism has two effects on wage. One pushes wage upward because the altruistic worker is less sensitive to the monetary payments from the subject-to-altruism Principal. Another pushes wage downward due to self-compensation, resulted from the partial internalization if the Principal's utility.

Establishing altruistic relation is endogenous in the model: the Agent chooses whether to accept an offer proposed by the subject-to-altruism Principal or an offer from another Principal. The conditions for establishing the altruistic relation are obtained. It is shown that higher uncertainty about Agent's abilities, higher importance of the future and lower Agent's altruism can prevent from establishing the altruistic relation. It means, in particular, that the altruistic Agent either establishes the non-altruistic relation first and then switches to the altruistic one or establishes the altruistic relation and never switches. The paper also shows that ambitions resulted from pushing himself too hard makes it impossible to establish altruistic relation in the future whereas laziness is safe for it.

${ }^{1}$ The Economist, Apr. 7, $2004-" T h e$ gift relationship" 
Interestingly, the paper provides an example of the "winner's blessing".

The model of the paper allows different interpretations. One is labor contract between friends or relatives. Another is local public good procurement by the insider producer which internalizes benefits from the produced public good (unlike the outside provider). The case of the agent possessing financial stake gives one more application of the model. The first interpretation is used throughout the paper for illustrative purposes.

\section{Related Literature}

My paper contributes to the quite new stream of literature which can be called "behavior agency theory".

Altruism is a psychological feature of the human beings. Behavioral economics stresses the role of psychology in economic interactions and personal decision making. The interaction in the workplace is a person-to-person interaction, so psychological features can be important.

Rotemberg (1994) considers the 2-stage interaction of the two economic agents in which they choose their degrees of altruism with respect to each other and then play the $2 * 2$ Prisoner's Dilemma. Importantly, the paper shows that altruism can emerge endogenously when the Agent is given the opportunity to choose between altruism and selfishness. The paper also shows that altruism emerges in the equilibrium when the workers are paid on the basis of the joint output. In the traditional models, the joint performance evaluation leads to moral hazard in teams. As a result, the workers exert suboptimal effort. However, in the presence of altruism effort levels are higher and workers are better off. Rotemberg argues that altruism may in fact emerge through the means of socializing between the workers.

A number of contributions on the role of psychology in the workplace relation has been made recently. They, however, focus on envy or spitefulness. The literature may be divided into two groups. One studies different aspects of envy of the Agent with respect to the Principal. Another considers multi-Agent setup with Agents envious (e.g. inequality-averse) with respect to each other.

Itoh (2004) considers the Principal-Agent relation with other-regarding preferences from the Agent as well as from the Principal side. The Agent is assumed to be spiteful with respect to the Principal. The paper finds that the Principal's payoff decreases as Agent's spitefulness increases whereas wage increases with spitefulness, which is quite natural. More subtle results is that in the multi-agent case with agents spiteful to each other the Principal can exploit the other-regarding nature of the Agents by designing the appropriate contract.

Dur and Glazer (2004) consider the model with Agent which is envious to the Principal. The paper shows there are two effects of envy affecting effort in different directions. Envy reduces effort for a given incentive but in equilibrium the Principal provides higher incentives. So, the overall effect is not clear. It depends on risk-aversion and properties of the envy functional form. In some cases envy can make profit-sharing optimal even if effort is contractible.

Bartling and von Siemens (2006) consider the model with 2 agents working in team. Wages are conditioned on team output. The workers are inequityaverse and envious to each other. The paper concludes that envious agents suffer if other agents receive higher wage due to random performance shocks. 
The necessary compensation for expected envy renders incentive provision more expensive, which generates a tendency towards flat-wage contracts.

The analysis of Goel and Thakor (2003) demonstrates difference in optimal contract design for envious agents and shows envy has two impacts affecting in different directions.

An interesting example is provided by Grund and Sliwka (2005). The paper considers other-regarding preferences in the framework of tournaments. It is argued that the inequality averse agents dislike the inherent inequality of the tournament, so they have to be compensated for it through increase in wage. On the other hand, this inequality aversion creates extra incentive to exert effort.

A critical survey of the theories of extrinsic and intrinsic incentives and evidence are provided by Fehr and Falk (2002). It is clear that intrinsic and extrinsic motivation interact in the non-trivial way, in particular the literature shows that intrinsic motivation can be crowded-out by extrinsic motivator such as high-powered wage scheme - see the model in Bénabou and Tirole (2006) in which extrinsic motivation crowds-out intrinsic incentives because it influences the agent's self-respect or social reputation. Sliwka (2007) provides a model in which high-powered extrinsic incentives signal about dishonesty acceptance in social norms and hinder workers' intrinsic incentives. The experiment in Falk and Kosfeld (2006) shows that a principals decision to control has a negative impact on the agents motivation. Interestingly, unlike the literature cited above, this paper provides an example where intrinsic motivator lessens extrinsic motivation.

\section{The model setup}

Consider the Principal-Agent two-period relationship as in Holmstrom (1999) career concerns model. Workers (agents) are characterized by their type (skill or talent) $\theta$. Assume for simplicity that $\theta$ is not known neither by the Agent nor by the Principal. There is common prior belief that $\theta$ is normally distributed:

$$
\theta \sim N\left(\bar{\theta}, \sigma_{\theta}^{2}\right)
$$

In each period $j=1,2$ the Agent chooses effort level $a_{j}$. Output level is determined by

$$
y_{j}=\theta+a_{j}+\varepsilon_{j}
$$

Output noises $\varepsilon_{j}$ are identically distributed, independent from each other and from $\theta$. Each of them is normally distributed with mean 0 and variance $\sigma_{\varepsilon}^{2}$ :

$$
\varepsilon \sim N\left(0, \sigma_{\varepsilon}^{2}\right)
$$

Effort is costly. The cost function $C(a)$ is assumed to be increasing, convex, with no cost at zero effort:

$$
C^{\prime}(a) \geq 0, \quad C^{\prime \prime}(a) \geq 0, \quad C(0)=0, \quad C^{\prime}(0)=0 .
$$

Assume that there are many workers and managers and that there is reciprocal altruism in only one pair worker-manager. It means that there is one particular worker who is altruistic to one particular manager and vice versa, whereas there is no altruism in any other worker-manager pair. 
To justify such exclusivity of the altruistic relation consider an example of two friends, one of them is an entrepreneur, looking for an engineer for his enterprise, the other is an engineer. There are many other enterprises which need engineers to hire and many other engineers seeking for the job. There is no friendship relation in any other pair entrepreneur-engineer. Of course, one can think of another form of a social relationship.

So, the two altruistic persons have an opportunity of establishing the altruistic relation, but each of them also has an outside option to establish the non-altruistic relation with someone else. The existence of the outside option captures the fact that altruism is not universal - the person is not altruistic to everyone.

The timing is as follows. At the beginning of the first period the managers offer salaries to workers. The salaries may be worker-specific; take-it-or-leave-it offers are made. After this, each worker chooses a manager, whose proposal he accepts. The output produced by each worker in the first period is observed by everyone. On the basis of this observation, all workers and managers make an inference on the workers' type. At the beginning of the second period all the managers again make worker-specific salary offers, then each worker chooses manager.

The own utilities of Agent (worker) and Manager obtained in each period are:

$$
\begin{gathered}
u_{j}=w_{j}-C\left(a_{j}\right) \\
v_{j}=y_{j}-w_{j}
\end{gathered}
$$

The Manager's and Worker's total (social) utilities obtained in period $j$ are given by

$$
\begin{aligned}
U_{j} & =u_{j}+\beta v_{j}=w_{A_{j}}-C\left(a_{A_{j}}\right)+\beta\left(y_{M_{j}}-w_{M_{j}}\right) \\
V_{j} & =v_{j}+\alpha u_{j}=y_{M_{j}}-w_{M_{j}}+\alpha\left(w_{A_{j}}-C\left(a_{A_{j}}\right)\right)
\end{aligned}
$$

where the parameters $\alpha$ and $\beta(0 \leq \alpha, \beta \leq 1)$ will be referred to as altruism parameters. The altruistic person partially internalizes the gains of another person. It is assumed that the altruism parameters $\alpha$ and $\beta$ are known to everybody.

The following notation is used: $y_{M_{j}}$ is output obtained by Manager, $w_{M_{j}}$ is salary paid by Manager, $w_{A_{j}}$ is salary received by Agent, $a_{A_{j}}$ is effort level exerted by Agent.

Note that in general there are two possibilities: 1) altruistic worker works for altruistic manager or 2) altruistic Worker works for another manager and another worker works for altruistic Manager. The first is said be the altruistic relation. The second is said to be the non-altruistic relation. In the analysis of the altruistic relation the latter is considered as an outside option for the former.

In the first (altruistic) case $w_{M_{j}}=w_{A_{j}}$ and we will refer to this salary level as "altruistic salary" and denote it as $w_{j}^{A}$. The effort exerted by the Agent in period $j$ is used to produce output. This effort level will be referred to as "altruistic effort" and denoted as $a_{j}^{A}$. Output obtained by the Manager is referred to as "altruistic output".

In the second case, when the altruistic relation is not established, the Manager hires another worker and the Worker goes to another manager. For the 
two pairs the non-altruistic relation is established. It is clear that the relations established are totally equal, which means that salaries paid by the Manager (to another worker) and received by the Agent (from another manager) are be at the same level $^{2}$. This is true also for effort level and output. We will denote corresponding levels as $w_{j}^{N A}, a_{j}^{N A}$ and $y_{j}^{N A}$. They will be referred to as "non-altruistic" levels.

The symbols without indexes $A$ or $N A$ will be used to denote the equilibrium values of parameters.

The two-periods utility for each agent is given by

$$
U=U_{1}+\delta U_{2} \quad\left(V=V_{1}+\delta V_{2}\right)
$$

where $\delta>0$ is the relative value of the 2 -nd period utility with respect to the 1 -st period utility. Notice that $\delta \geq 1$ is possible as well as $\delta<1$. The latter is the case in the model with time discounting. The former is reasonable if, for example, the first period (trial, untenured job) is shorter than the second period (permanent, tenured job).

The Managers and the Workers maximize their respective two-period total utilities.

\section{$3 \quad$ Efficiency}

The Pareto-optimal level of effort is given by

Lemma 1. The First-Best level of effort is determined by

$$
C^{\prime}\left(a^{F B}\right)=1
$$

and doesn't depend on the level of altruism.

Proof is given in the Appendix.

Notice that the First-Best effort level doesn't depend on the altruism parameters. In particular, it coincides with the First Best for the "standard" non-altruistic relation. So, if selfish utilities are considered instead of "social" utilities, the First Best effort will be the same.

It is also interesting to note that the utility possibility set under the optimal effort level is given by:

$$
(1-\alpha) U_{A}+(1-\beta) U_{M} \leq(1-\alpha \beta)\left(a^{F B}+\theta-C\left(a^{F B}\right)\right)
$$

This means, in particular, that the sum of utilities $U+V$ may achieve any level through monetary transfers (wage). For example, if the Manager is more altruistic than the Worker, $\alpha>\beta$, then increasing the wage to $+\infty$ increases also the sum $U+V$ to $+\infty$. Note that in this case $U \rightarrow+\infty$ and $V \rightarrow-\infty$. This makes the utilitarian First Best analysis in the presence of the altruism somewhat ambiguous.

\footnotetext{
${ }^{2}$ but the altruistic worker would receive the salary from another manager.
} 


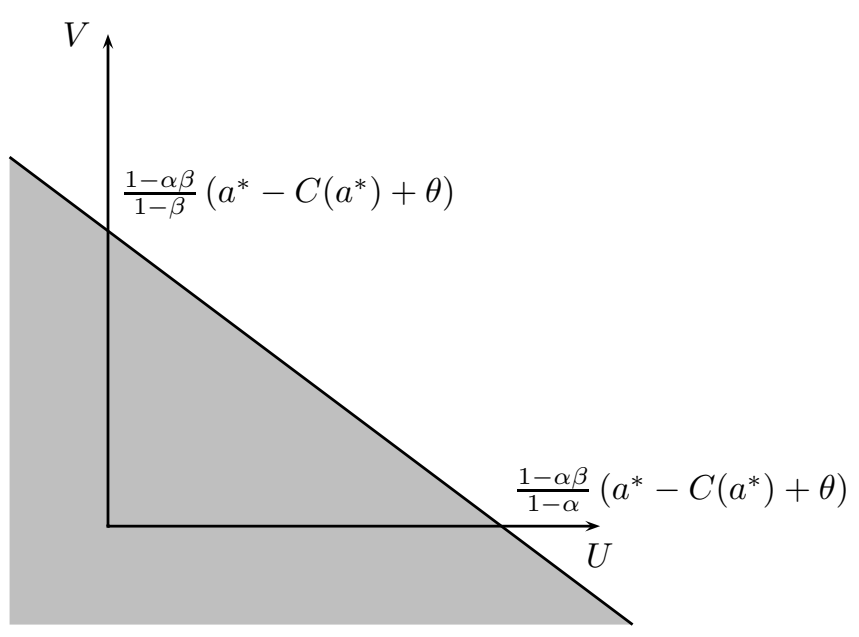

Figure 1: Utility Possibility set

\section{Equilibrium}

To find an equilibrium of the 2-stage game, we proceed backward.

At the beginning of the first period, there is the prior belief for distribution of worker talent. As stated above,

$$
\theta \sim N\left(\bar{\theta}, \sigma_{\theta}^{2}\right)
$$

At the end of the 1-st period, the output is observed, and update for the distribution of the talent parameter $\theta$ is made. It will be shown below that this update doesn't depend on the type of the relation, established in the 1-st period (altruistic or not).

\subsection{Period 2}

First, beliefs on the Worker talent are updated. Since the worker doesn't know his type at the beginning of the first period, the effort choice and the Manager's belief don't depend on $\theta$. The following standard lemma describes how the beliefs are updated.

Lemma 2. Suppose that the Manager believes that the 1-st period effort level is $a_{1}^{\mu}$ while the actual effort is $a_{1}$. Then:

1. The Managers update their beliefs on the distribution of the worker's type $\theta$ according to the formula:

$$
\begin{gathered}
\theta \sim N\left(\overline{\theta_{2}^{M}}, \sigma_{\theta}^{2}\right) \\
\overline{\theta_{2}^{M}}=\lambda\left(y_{1}-a_{1}^{\mu}\right)+(1-\lambda) \bar{\theta}
\end{gathered}
$$

2. The Worker updates his belief on the distribution of his own type $\theta$ according to the formula:

$$
\theta \sim N\left(\overline{\theta_{2}^{A}}, \sigma_{\theta}^{2}\right)
$$




$$
\overline{\theta_{2}^{A}}=\lambda\left(y_{1}-a_{1}\right)+(1-\lambda) \bar{\theta}
$$

where

$$
\lambda=\frac{\sigma_{\theta}^{2}}{\sigma_{\theta}^{2}+\sigma_{\varepsilon}^{2}}
$$

Proof is standard. See, e.g. Holmstrom (1999).

The parameter $\lambda$ may be interpreted as the relative uncertainty of the worker's talent. There are two sources of the uncertainty in the model - worker's talent and noise in output. The higher the variance of the distribution of talent $\theta$, i.e. the higher the uncertainty on $\theta$, the higher is $\lambda$. And $\lambda$ grows up to 1 as the uncertainty on $\theta$ increases. When the worker's talent is known for sure, the relative uncertainty of talent $\lambda=0$.

Notice that the worker belief update doesn't depend on the actually exerted effort. In fact, any increase in effort results in the same increase in output, so the difference $y_{1}-a_{1}$ in formula (2) is nor affected. If $\theta$ is worker's true type then formula (2) may be rewritten as

$$
\overline{\theta_{2}^{A}}=\lambda\left(\theta+a_{1}+\varepsilon_{1}-a_{1}\right)+(1-\lambda) \bar{\theta}=\lambda\left(\theta+\varepsilon_{1}\right)+(1-\lambda) \bar{\theta}
$$

Notice also that if the worker exerts higher (lower) effort, compared to what he has been expected, his own update isn't affected, but the Manager's update is.

Consider now effort choice and wage setup in the second period. There are two possibilities: to establish altruistic or non-altruistic relation.

\section{The Non-altruistic relation}

If the non-altruistic relation is established, the altruistic Agent's program is

$$
\max _{a_{2}}\left\{-C\left(a_{2}\right)+w_{2}^{N A}+\beta v_{2}^{N A}\right\}
$$

The altruistic worker still cares about the Manager's wealth, even though they are not working together - this is why we have the term $\beta v_{2}^{N A}$, where $v_{2}^{N A}$ is the utility, obtained by the the Manager, when he hires a non-altruistic worker. Evidently, this term doesn't depend on the altruistic Worker's decision.

The non-altruistic workers' program is the same with $\beta=0$.

The non-altruistic effort level (exerted by non-altruistic workers as well as by the Altruistic Worker) is

$$
a_{2}^{N A}=0
$$

The non-altruistic Managers' expected utility is

$$
V_{2}^{N A}=E_{2}^{M}\left[\widetilde{y}_{2}-\widetilde{w}_{2}\right]=E_{2}^{M}\left[\theta+a_{2}^{N A}+\varepsilon_{2}-\widetilde{w}_{2}\right]=\overline{\theta_{2}^{M}}-\widetilde{w}_{2}
$$

Here $\widetilde{y}_{2}$ is the output obtained by manager and $\widetilde{w}_{2}$ is the wage, paid to the Worker by one of the managers. Since there is competition between managers à-la Bertrand, it should be $E_{2}^{M}\left[V_{2}^{N A}\right]=0$, so that the non-altruistic wage offered to each worker is

$$
w_{2}^{N A}=\overline{\theta_{2}^{M}}
$$

The utility levels obtained under the non-altruistic relationship are:

$$
V_{2}^{N A}=0+\alpha \overline{\theta_{2}^{M}}
$$




$$
U_{2}^{N A}=\overline{\theta_{2}^{M}}+\beta \cdot 0
$$

The altruistic Manager gets extra utility due to positive own utility of the Worker.

Notice that the Managers' belief (not the Worker's!) determines the worker's utility in (5).

\section{The Altruistic Relation}

The Agent's expected utility level obtained in the 2-nd period is

$$
\begin{aligned}
U_{2} & =E_{2}^{A}\left[w_{2}^{A}-C\left(a_{2}^{A}\right)+\beta\left(y_{2}^{A}-w_{2}^{A}\right)\right]= \\
& =E_{2}^{A}\left[w_{2}^{A}-C\left(a_{2}^{A}\right)+\beta\left(\theta+a_{2}^{A}+\varepsilon_{2}-w_{2}^{A}\right)\right]= \\
& =\beta a_{2}^{A}-C\left(a_{2}^{A}\right)+(1-\beta) w_{2}^{A}+\beta \overline{\theta_{2}^{A}}
\end{aligned}
$$

The Agent's program is then

$$
\max _{a_{2}}\left\{\beta a_{2}-C\left(a_{2}\right)+(1-\beta) w_{2}^{A}+\beta \overline{\theta_{2}^{A}}\right\}
$$

Again, as for the non-altruistic relation, the solution doesn't depend on the wage.

The altruistic effort level in the second period is given by

$$
C^{\prime}\left(a_{2}^{A}\right)=\beta
$$

So, we obtain the following characterization of the altruistic relation in the 2 -nd period, which is also true for the one-period interaction.

Lemma 3. In the last period (or in the one-period interaction), the effort is given by $(6)$.

Effort increases with altruism.

Notice that the effort level doesn't depend on the inference on the type $\theta$.

Claim 1. In the last period, the Altruistic relation is more efficient than the non-Altruistic one.

Indeed, the effort of the altruistic worker, determined by (6), is closer to the optimal effort $\left(C^{\prime}\left(a^{F B}\right)=1\right)$ than the effort of the non-altruistic worker $\left(a_{2}^{N A}=0\right)$. Still, the altruistic effort level is suboptimal.

Utilities obtained under the altruistic relation by the Worker and by the Manager correspondingly are given by

$$
\begin{aligned}
& U_{2}^{A}=w_{2}^{A}-C\left(a_{2}^{A}\right)+\beta\left(E^{A} y_{2}^{A}-w_{2}^{A}\right)=w_{2}^{A}-C\left(a_{2}^{A}\right)+\beta\left(\overline{\theta_{2}^{A}}+a_{2}^{A}-w_{2}^{A}\right) \\
& V_{2}^{A}=E^{M} y_{2}^{A}-w_{2}^{A}+\alpha\left(w_{2}^{A}-C\left(a_{2}^{A}\right)\right)=\overline{\theta_{2}^{M}}+a_{2}^{A}-(1-\alpha) w_{2}^{A}-\alpha C\left(a_{2}^{A}\right)
\end{aligned}
$$

Consider now the possibility of establishing the altruistic relation in the second period on the equilibrium path. 
Lemma 4. On the equilibrium path, $\overline{\theta_{2}^{A}}=\overline{\theta_{2}^{M}}\left(=\overline{\theta_{2}}\right)$ and in the 2-nd (last) period:

1. The altruistic relation is always established.

2. The worker gets utility $U_{2}=\overline{\theta_{2}}$

and receives wage

$$
w_{2}^{A}=\overline{\theta_{2}}-\frac{\beta}{1-\beta} a_{2}^{A}+\frac{1}{1-\beta} C\left(a_{2}^{A}\right)
$$

Proof is given in the Appendix.

From Claim 1 we can conclude that if the beliefs of the worker and the managers coincide, then altruism always leads to the efficiency improvement in the 2-nd period.

For the analysis of possible deviations from the Equilibrium path it is important to consider the period 2 decision making when beliefs of the Worker and the managers on the worker's type are different.

Lemma 5. If worker's effort in the 1-st period $a_{1}$ differs from the managers beliefs on the effort, conditioned on choice of the Altruistic (non-Altruistic) relation in the 2-nd period $A_{L}\left(A_{H}\right)$, and, consequently, beliefs of the worker and the managers on the worker type distribution after the 1-st period are different, then in period 2:

1. The altruistic relation is established iff

$$
a_{1} \leq A_{L}+\frac{A_{H}-A_{L}}{\beta}
$$

2. The non-altruistic relation is established iff

$$
a_{1} \geq A_{L}+\frac{A_{H}-A_{L}}{\beta}=A_{H}+\left(\frac{1}{\beta}-1\right)\left(A_{H}-A_{L}\right)
$$

3. The utilities obtained by the Worker under altruistic and non-altruistic relation are

$$
\begin{gathered}
U_{2}^{A}=\bar{\theta}+\lambda a_{1}-\lambda\left((1-\beta) A_{L}+\beta a_{1}\right) \\
U_{2}^{N A}=\bar{\theta}+\lambda a_{1}-\lambda A_{H}
\end{gathered}
$$

Proof is given in the Appendix.

Notice that the Utilities obtained by the Worker may be rewritten as $U_{2}^{A}=$ $(1-\beta) \overline{\theta_{2 H}^{M}}+\beta \overline{\theta_{2}^{A}}$ and $U_{2}^{N A}=\overline{\theta_{2 L}^{M}}$, where $\overline{\theta_{2 H}^{M}}$ and $\overline{\theta_{2 L}^{M}}$ are the Managers' updated beliefs, conditional on the relation type choice in the 2-nd period.

The first case in the lemma appears after a first period downward deviation or not too high upward deviation. It appears, for example, when the worker is lazy at the 1-st period and exerts less effort than it is expected by the Manager. In this case altruistic relation at the 2-nd period is established.

The second case appears after high enough upward deviation, i.e. when the worker exerts much more effort than he is expected to. He did this on the purpose to cheat the market by making the others believe he is very productive (or talented). In this case he becomes ambitious in the 2-nd period and wants higher wage. He knows that any manager gets less output than he expects but pays high wage according to the expectation. The worker sacrifices the losses 
that the subject-to altruism Manager might suffer and doesn't sacrifice from the losses of the non-altruistic ones. This forces the worker to establish the non-altruistic relationship. In other words, the worker prefers to cheat anyone but his friend Manager. In this case altruistic relation is severed.

Put differently, downward or laziness deviation is relatively safe to hold friends working together. Whereas high upward deviation makes the Worker ambitious and severs altruistic relation. This means, for example, that if altruistic relation has some additional value for the worker, not related to the production process, it is more likely that he would be lazy rather than overworking.

\section{$4.2 \quad$ Period 1}

The key difference from the period- 2 interaction is that in the period- 1 the career concerns are presented. The decision, made in the period 1 (i.e. effort level), affects the expected utility for the second period through updating the Principal's beliefs on the worker's type $\theta$, based on the observed period- 1 output. So, when making the decision (i.e. choosing the effort), the worker cares not only about period-1 utility, but also about the expected utility for the period 2 .

The model may be summarized by the following sketch of the game tree (see Figure 2).

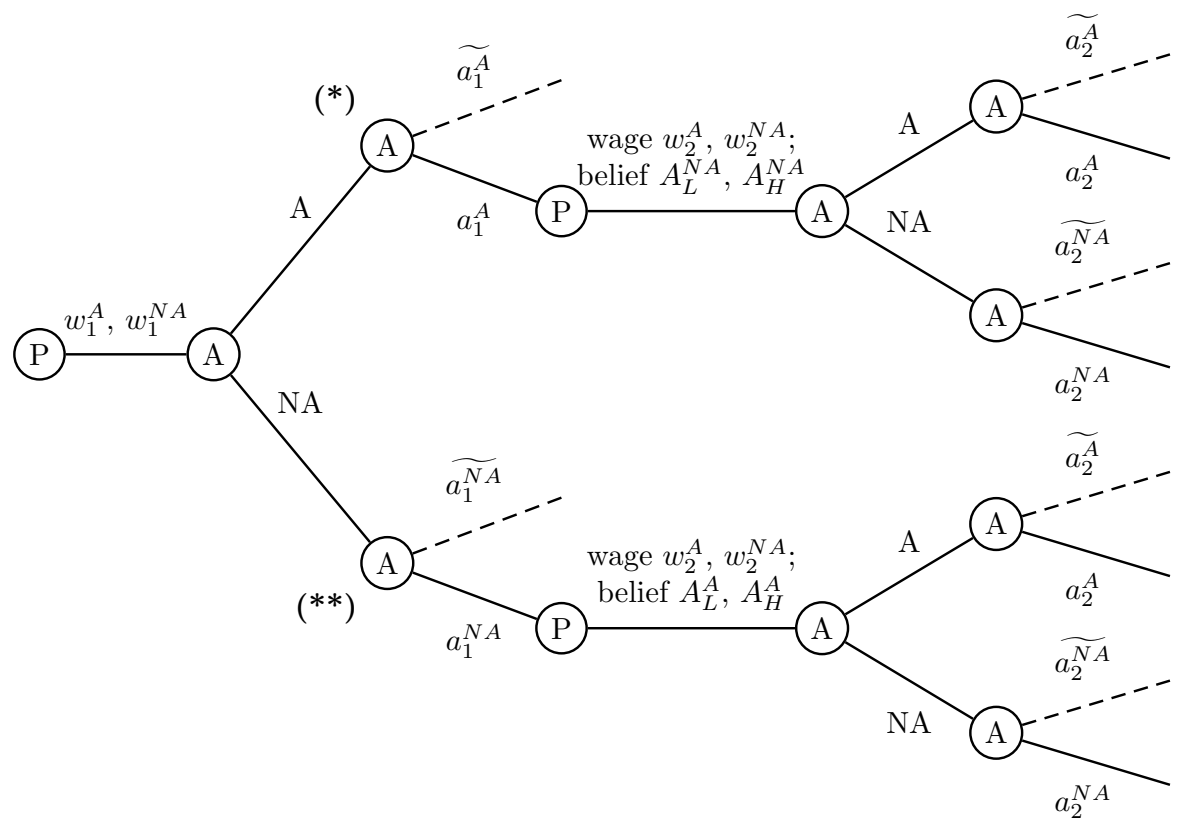

Figure 2: Sketch of the game tree

Comment to the game tree. Notation: P - Principal(s) move, A - Agent move. Efforts with tilde and dashed nodes mean deviation from equilibrium effort choice. Nature moves (output noise realizations) are not shown to keep the tree simpler.

Importantly, the beliefs on the first period effort are based on the choice of the relation type in the second period. Consequently, the 2-nd period wage offers $\left(w_{2}^{A}, w_{2}^{N A}\right)$ are different for altruistic and non-altruistic relation choice in 
the 2-nd period. It is easier to think that the Principals observe the relation type choice made by the worker in the 2 -nd period (i.e. whether the worker goes to the subject-to-Altruism Principal or to another one) and after this update their beliefs on the 1-st period effort and afterwards on the worker's type.

Proposition 1. There exists pure strategies Perfect Bayesian Equilibrium which is characterized by the following efforts and beliefs:

1. The beliefs on the effort choice in 1-st period are conditional on the relation type chosen in the 2-nd period;

2. $a_{L}^{A}, a_{L}^{N A}$ are the actually chosen efforts under the altruistic and nonaltruistic relation in the 1-st period correspondingly; these efforts are conditional on the choice of the altruistic relation in the 2-nd period. These efforts are determined by

$$
C^{\prime}\left(a_{L}^{N A}\right)=(1-\beta) \delta \lambda \quad C^{\prime}\left(a_{L}^{A}\right)=(1-\beta) \delta \lambda+\beta
$$

3. $a_{H}^{A}, a_{H}^{N A}$ are the potential efforts choices (which are never realized, they only support equilibrium) under the altruistic and non-altruistic relation in the 1-st period correspondingly; these efforts are conditional on the choice of the non-altruistic relation in the 2-nd period. They are determined by

$$
C^{\prime}\left(a_{H}^{N A}\right)=\delta \lambda \quad C^{\prime}\left(a_{H}^{A}\right)=\delta \lambda+\beta
$$

4. Managers' beliefs $A_{\bullet}^{\bullet}$ coincide with the effort levels.

Proof is given in the Appendix.

Notice that the worker may exert an overoptimal effort in the 1-st period due to high career concerns. The result is similar to one obtained by Holmstrom (1999). However, in the presence of altruism, the excessive effort is softened; whereas in the case of underprovision of effort, altruism incentivizes the worker. So, effort is always closer to the optimum in the presence of altruism. The last step in the analysis is the choice of the relation type for the 1-st period. It is characterized by the following proposition.

Proposition 2. 1. The altruistic relation is established in the 1-st period iff it's more efficient than the non-altruistic relation, i.e. iff

$$
\Delta C \leq \Delta a
$$

where

$$
\Delta C=C\left(a_{L}^{A}\right)-C\left(a_{L}^{N A}\right) \quad \Delta a=a_{L}^{A}-a_{L}^{N A}
$$

2. For a given $\beta$, the Altruistic relation is established iff

$$
\delta \lambda<r(\beta)
$$

where $r(\beta)$ is an increasing function,

$$
r(0)=1, \quad r(\beta)>1 \text { for } \beta>0, \quad \lim _{\beta \rightarrow 1}=+\infty
$$


Proof is given in the Appendix.

Figure 3 gives an illustration of the Proposition 2.

The straightforward corollary of the Proposition 2 is that the higher values of the relative importance of the 2-nd period $(\delta)$, or the relative uncertainty of the worker's talent $(\lambda)$ limit establishing the altruistic relation in the 1-st period. Higher altruism is conducive to altruistic relation in the 1-nd period.

Notice that, despite the Agent internalizes only a part of the Principal's utility, this is enough to achieve the efficient outcome in the worker's choice of the relation type (though the choice is binary).

\section{$5 \quad$ Discussion and Applications}

This section complements previous formal analysis by developing economic intuition on the impact of altruism and discusses applications of the model.

Recall that when altruistic relation is established at the 1-st period, effort in equilibrium is determined by (see proposition 1)

$$
C^{\prime}(a)=(1-\beta) \delta \lambda+\beta
$$

Compare this with effort chosen by the non-altruistic worker $(\beta=0)$ :

$$
C^{\prime}(a)=\delta \lambda
$$

and effort chosen by altruistic worker under the non-altruistic relation in the 1-st period:

$$
C^{\prime}(a)=(1-\beta) \delta \lambda
$$

The multiplier $(1-\beta)$ reflects reduced career concerns. It appears only for the altruistic worker independently of the type of the relation established in the 1-st period and is due to anticipation of establishing altruistic relation in the future (2-nd period). The item $(+\beta)$ appears only if altruistic relation is currently established and reflects a stimulating effect of it.

It is important to distinguish between current and anticipated altruism as they have different nature and different impact on effort.

The two types of altruism are presented simultaneously when the altruistic relation is established at the 1-st period. Indeed, in the 2 -nd period the altruistic relation is always established (see Proposition 4) so the anticipated altruism is at work. The current altruism is clearly presented since current relation is altruistic.

It is well possible that only anticipated altruism is presented. This is the case when the non-altruistic relation is established in the 1-st period. Finally, in the 2-nd period only current altruism is presented since altruistic relation is always established and there is no future in the model.

However, in the real world the future always exists and the altruistic relation is expected to be established, perhaps with some probability, so the anticipated altruism is always at work. This is important to be noticed because current altruism is somewhat evident whereas anticipated altruism is more subtle but has an influence as well. 
Another way to illustrate the impact of altruism is to decompose the effort choice in the 1 -st period for the altruistic relation in (9) in the following way:

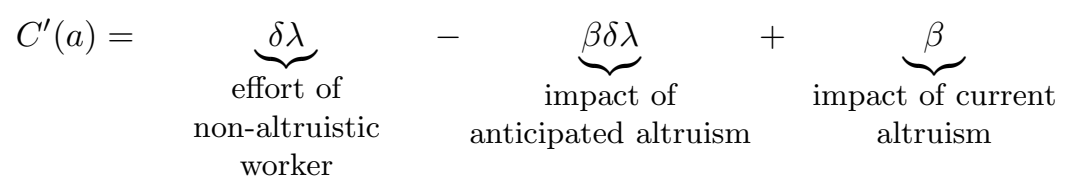

The term $\delta \lambda$ represents the career concerns incentive, as in the model with selfish workers. Second term $\beta \delta \lambda$ shows that the career concerns incentive is weakened for the altruistic worker. Finally, the third term $\beta$ represents the incentive emerging from the intrinsic motivation of the worker.

Which altruism impact is stronger depends on the relative uncertainty of worker's skills $\lambda$ and relative importance of the future $\delta$. The following claim follows directly from equation (10) and Proposition 2.

Claim 2. 1. Higher levels of relative uncertainty about skills $\lambda$, higher relative importance of the future $\delta$ and higher altruism level $\beta$ make anticipated altruism stronger.

2. Only higher altruism level $\beta$ makes current altruism stronger.

3. Current altruism increases effort whereas anticipated altruism decreases it.

4. Anticipated altruism is dominant iff $\delta \lambda>1$, i.e. the effort of the altruistic worker is lower than that of the non-altruistic one. Altruistic or nonaltruistic relation can be established. Effort decreases with altruism but has a jump when the type of the relation changes (see Figure 4- Left).

5. Current altruism is dominant iff $\delta \lambda<1$, i.e. effort of the altruistic worker is higher than that of the non-altruistic one. Altruistic relation is always established. Effort increases with altruism (see Figure 4-Right).

The Claim states that the dominance of one of the altruism types is determined by the career concerns $\delta \lambda$, rather than by altruism. The impact of altruism is to magnify the prevalence of one of the altruism types. For instance, if the anticipated altruism dominates, the higher altruism makes its dominance over the current altruism more pronounced. Consequently, in this case effort decreases with altruism. If only anticipated altruism is presented, effort decreases with altruism due to the magnification effect as well.

Figure 3 illustrates Claim 2 and Proposition 2. The horizontal line $\delta \lambda=$ 1 separates the regions of the current altruism and the anticipated altruism dominance as followed from Claim 2. The area of the non-altruistic relation is shaded. The separating line between the altruistic and non-altruistic relation areas is determined by the function $r(\beta)$ from Proposition 2. Interestingly, the optimal effort level is achieved only under the non-altruistic relationship.

Figure 4 may be considered as projections of figure 3. It shows effort as function of the altruism parameter $\beta$. When $\delta \lambda>1$ effort is piece-wise decreasing but there is a jump corresponding to the switching from non-altruistic to altruistic relationship. So, in general effort is not a monotone function of altruism. When $\delta \lambda<1$ effort increases with $\beta$.

Now we turn to the analysis of how altruism influences wages. 


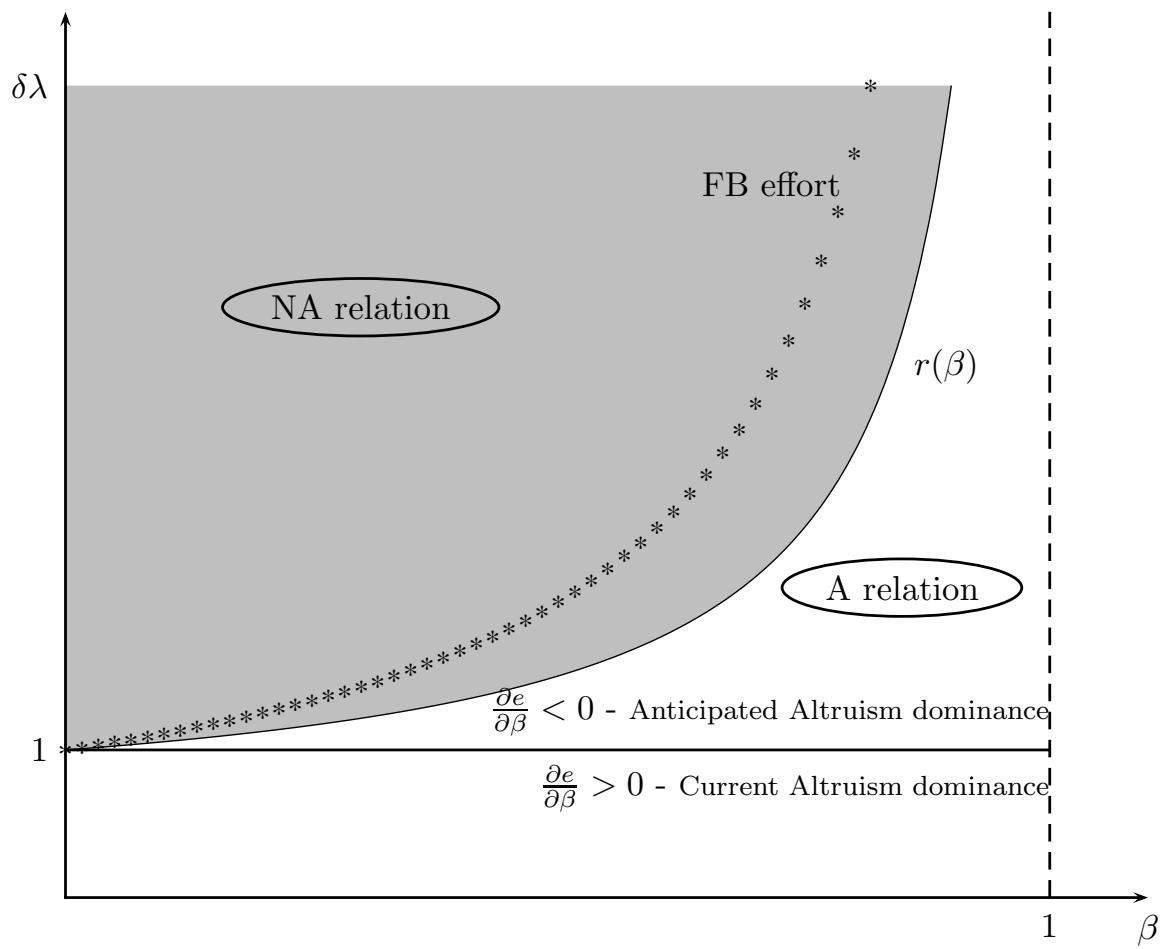

Figure 3: Type of relationship and dominant type of altruism.

Claim 3. A. There are two effects related to monetary compensation from the Principal which is subject to altruism:

1. Decreased sensitivity to monetary payments: the altruistic Agent is harder to attract by monetary payments from subject-to-altruism Principal.

2. "self-compensation": the altruistic Agent gets extra utility due to extra output resulted from extra effort and requires less monetary compensation.

B. The two effects work in the opposite directions.

To prove the claim, point out that the difference in wages of altruistic and non-altruistic workers is given by the formula:

$$
\Delta w=\frac{\Delta C}{1-\beta}-\frac{\beta \Delta a}{1-\beta}
$$

where $\Delta w=w^{A}-w^{N A}, \Delta C=C\left(a^{A}\right)-C\left(a^{N A}\right), \Delta a=a^{A}-a^{N A}$

There is a natural interpretation of the wage difference formula (11). Increase in wage for the altruistic worker not only means an increase in his own utility (one-for-one) but also a decrease in the altruistic part of his utility related to Principal's wealth decrease (in $\beta$ for 1 proportion). So, the overall effect of wage increase for the worker's total utility is only $(1-\beta): 1$, i.e. to increase his utility by 1 unit (in order to compensate for effort disutility) the required wage increase is $\frac{1}{1-\beta}$ monetary units. As a result, to compensate marginal cost of extra effort in altruistic relation, the wage increase should be more than monetary equivalent of the cost. The more altruistic the worker is, the higher 

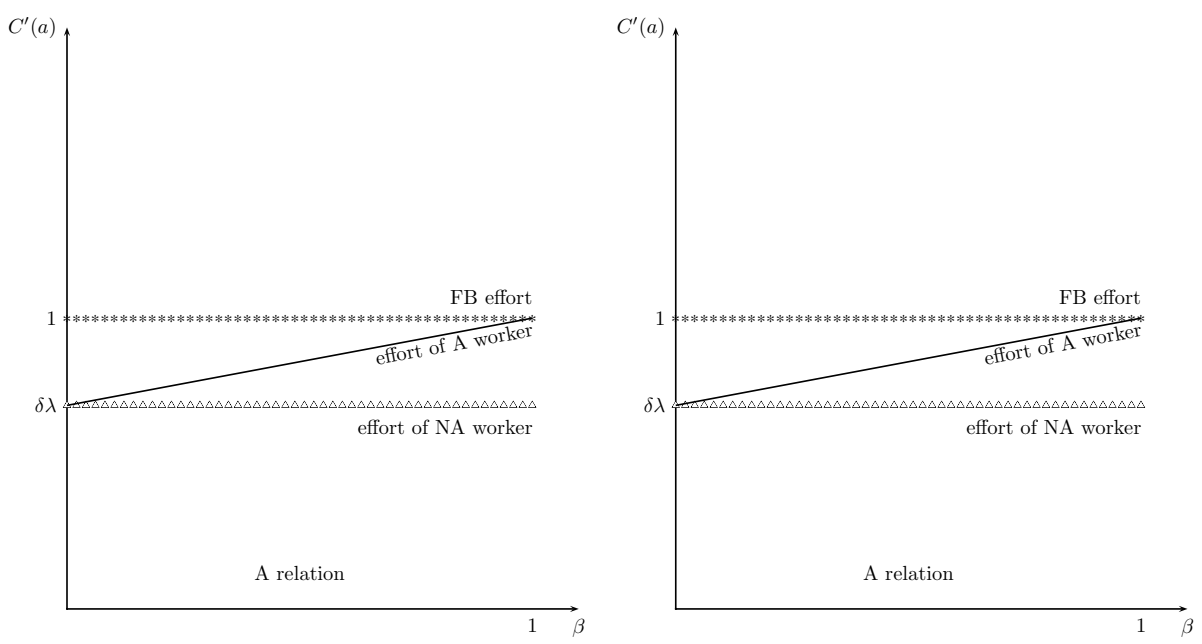

Figure 4: Effort level as function of altruism. Left - case $\delta \lambda>1$, Right - case $\delta \lambda<1$.

is difference between extra cost and extra payment required to compensate this cost. This is captured by the multiplier $\frac{1}{1-\beta}>1$ before the term $\Delta C$. This justifies the first point of the Claim.

On the other hand, due to altruism the share $\beta$ of the output increase resulted from the increased effort is incorporated into the worker's utility. So, there is partial "self-compensation" from the effort increase for the worker. Because of this, the Manager may compensate the Worker less through the monetary payments. Notice that the self-compensation is non-monetary effect. As a consequence, the more altruistic the worker is, the stronger is the self-compensation effect.

To sum up discussion related to wages, the altruistic worker's motivation comes not only from monetary rewards (as for the non-altruistic worker) but also through the non-monetary effect. Moreover, the effectiveness of the material rewards for the worker is lower for more altruistic worker. Consequently, due to decreasing sensitivity effect, more altruistic worker requires more monetary compensation to compensate the same disutility of effort. On the other hand, due to the self-compensation effect, more altruistic worker requires less monetary compensation for the same effort. the interaction of the two effects determines the wage the altruistic worker gets.

As it was already pointed out in the discussion after Proposition (5), if the worker exerts at the 1-st period more effort than he was expected, then he becomes ambitious and altruistic relation in period 2 may not be established. However, if the worker exerted less effort, altruistic relation still is going to be established in the future. So, ambitions hinder altruistic relation whereas laziness is safe to keep altruistic relation.

Another interesting feature of the model is "winner's blessing". Indeed, consider the two managers - one is subject to altruism another is not. They are competing for the worker to hire him. Each of them offers some wage to attract 
the worker. If the subject-to-altruism manager wins this competition, he will gain some rent. Indeed, his wage offer is such that by establishing altruistic relation the Worker will get the same utility (or slightly higher) as if under non-altruistic relation. However the outcome under altruistic relation is closer to the First Best compared to the non-altruistic one (see Proposition 2) and all the gains in overall utility goes to the winner (subject to altruism Manager).

As it was mentioned in the literature review, the model of the paper provides an example where intrinsic motivator (altruism) lessens extrinsic motivation (carrier concerns). Economical literature on motivation crowding as well as psychological, sociological and management literature is concentrated on crowding out of intrinsic motivation by extrinsic incentives. So, the paper provides an interesting example when crowding out effect goes different way.

The model extension to $\mathrm{T}$ periods with $T \geq 3$ may be considered as a sequence of two-period models with decreasing $\delta_{t}, \sigma_{\theta t}$ and as a consequence $\lambda_{t}$.

One can see from Proposition 2 that only two scenarios are possible: either the altruistic relation is established from the very beginning or the non-altruistic relation is be established first and at some moment it switches to the altruistic one. In the latter case, at the beginning effort decreases and it is below the nonaltruistic effort. At the moment of switching to the altruistic relation, effort jumps and may be higher or lower than the non-altruistic effort. Then effort decreases but more steadily than the non-altruistic effort. Starting from some point if not from the moment of jump it will be more than the non-altruistic one. This illustrates the impact of the two types of altruism. Under the nonaltruistic relation, only anticipated altruism is presented which pushes effort downward. After switching to the altruistic relation, current altruism emerges and pushes effort upward. Over time, effort declines due to decreasing career concerns. Anticipated altruism lessens the rate of declining because it lessens the cause of it.

The results of the paper can be applied to some issues in organizational economics. In particular, the following questions can be addressed. Should the Agent be informed about a possibility of working with subject to altruism Principal (his friend or family member)? If yes - at which moment? Consider the multiperiod setup. Assume that up to time $t_{1}$ the Agent works with some Principal $(\beta=0)$ and doesn't know about a possibility of establishing altruistic relation with the subject to altruism Principal (such possibility didn't exist or the organization designer didn't inform the Agent). So, up to the moment $t_{1}$ the Agent acts as the non-altruistic Agent. At time $t_{1}$ the Agent learns about possibility of establishing altruistic relation and may choose to change the Principal. Assume that $\delta_{t_{1}} \lambda_{t_{1}}$ is high enough so that the altruistic relation is not established. As a result, effort jumps down under the anticipated altruism influence. Over time, $\delta_{t} \lambda_{t}$ decreases and at some moment $t_{2}$ the Agent switches to the altruistic relation. At this moment effort jumps upward. Starting from this moment both altruism effects - anticipated and current are at work.

Figure 5 illustrates the dynamic of effort. It is clear that to avoid undesirable effort decline between $t_{1}$ and $t_{2}$ the Agent should be informed about the possibility of establishing altruistic relation not earlier than he actually wants to switch. 


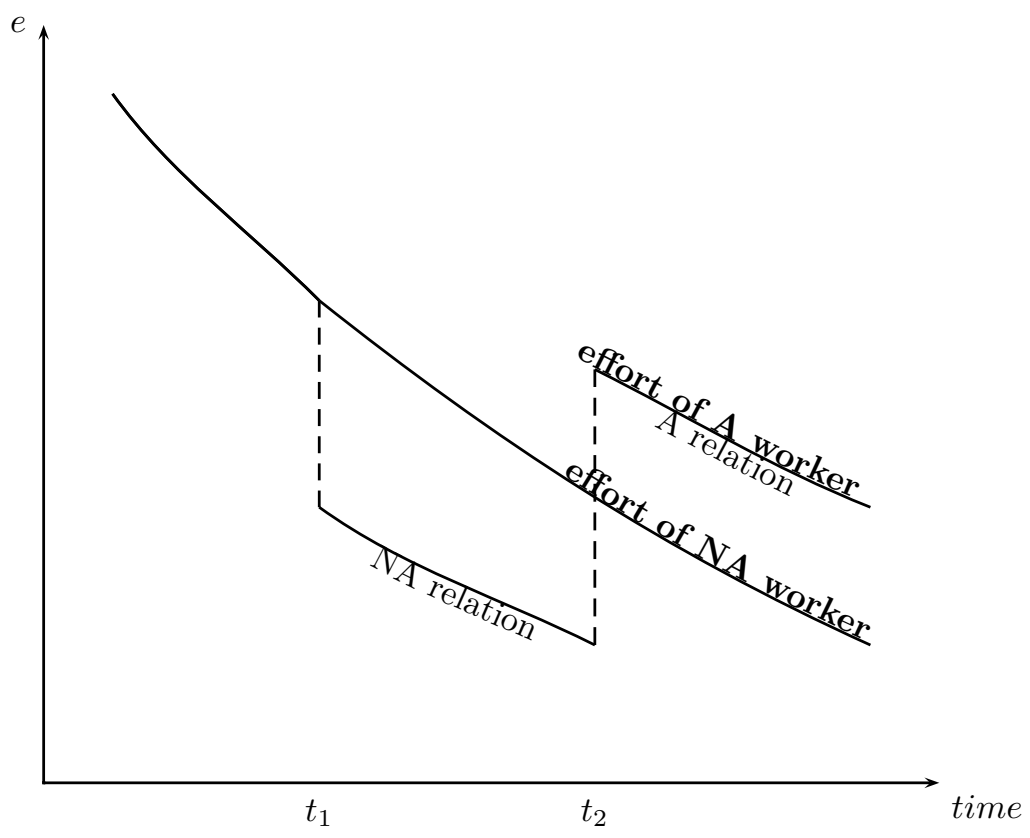

Figure 5: The effort dynamics

Throughout the paper the friends' labor contract interpretation was used. Let us turn to another interpretations - local public good provision.

Assume that local government (Principal) wants to hire a public good provider. After the public good is produced, it may be partially used by the producer if it resides in the location . Consider, for example, road (or roads network) construction. The road is long enough (or network is large) so that after it is constructed, the producer will use only part of it. There is also an alternative producer of the public good (the large nation-wide corporation) which is not the resident of the locality, so it will not use public good after it is produced. The local provider may produce the public good in some other location where it doesn't reside, so it won't use this public good after production. Provision of the public good is required for the two periods. The contract for the public good provision lasts only one period and specifies compensation from the local government to the provider. The provider is characterized by its skill $\theta$ and chooses effort level $a$. The quality of the public good is $\theta+a+\varepsilon$ where $\varepsilon$ is noise. The local public good provider uses share $\beta$ of the produced public good. The public funds are used to build roads and as well to produce other public goods. So, higher payments to the firm means lack of other public good provision. With this framework, the model analyzed in the paper may be applied. In particular, one can conclude that under high uncertainty $(\delta \lambda>1)$ the highest quality at the first period will be provided by the outside provider. However, if due to some reason the inside provider should be hired, in order to obtain higher quality of the public good, it shouldn't be too involved (i.e. $\beta$ shouldn't be too high - see Figure 4).

Another possible application of the model is the case when Agent possesses the financial stake. Consider a Manager who works at a firm and owns stakes 
of it. He may as well work at another firm, then the firm would have to hire another manager which is assumed not to have stakes of this particular firm. The higher monetary payments to the Manager will decrease firm profit and as a consequence dividends or even firm stock market value. So, the manager sacrifices to some extent monetary payments from the firm. So, the model developed in the paper can be applied to describe behavior of such a manager.

\section{Appendix}

\section{Proof of Lemma 1}

Proof. Consider one-period interaction with perfect information. Utilities are given by:

$U_{M}=y-w+\alpha(w-C(a))=\theta+a-w+\alpha(w-C(a))=a-\alpha C(a)-(1-\alpha) w+\theta$

$U_{A}=w-C(a)+\beta(y-w)=w-C(a)+\beta(\theta+a-w)=\beta a-C(a)+(1-\beta) w+\beta \theta$

The peculiarity of the model is that monetary redistribution through wage payment leads to change of the size of the pie (sum of the utilities). So, in the Pareto optimum not only effort level but also wage are to be determined.

Pareto-optimum is derived from the maximization program:

$$
\left\{\begin{array}{c}
\max _{a, w}\{\beta a-C(a)+(1-\beta) w+\beta \theta\} \\
\text { s.t. } a-\alpha C(a)-(1-\alpha) w+\theta \geq \overline{u_{M}}
\end{array}\right.
$$

The solution is

$$
\begin{gathered}
C^{\prime}\left(a^{F B}\right)=1 \\
w^{P O}=\frac{2-\alpha}{2(1-\alpha)}+\frac{\theta-\overline{u_{M}}}{1-\alpha}
\end{gathered}
$$

\section{Proof of Lemma 4.}

Proof. To establish the altruistic relation both Principal and Agent should be better off compared to the non-altruistic relation, i.e. two inequalities should hold:

$$
\left\{\begin{array}{l}
U_{2}^{A} \geq U_{2}^{N A} \\
V_{2}^{A} \geq V_{2}^{N A}
\end{array}\right.
$$

After substituting utility levels from (5), (4), (7) and (8) and rearranging we obtain:

$$
\begin{gathered}
w_{2}^{A} \geq \overline{\theta_{2}}+\frac{C\left(a_{2}^{A}\right)-\beta a_{2}^{A}}{1-\beta} \\
w_{2}^{A} \leq \overline{\theta_{2}}+\frac{a_{2}^{A}}{1-\alpha}-\frac{\alpha}{1-\alpha} C\left(a_{2}^{A}\right)
\end{gathered}
$$


For the two inequalities to hold simultaneously the right-hand side of 14 should be greater than the right-hand side of 13. After rearranging the terms we obtain the condition for establishing the altruistic relation:

$$
a_{2}^{A} \geq C\left(a_{2}^{A}\right)
$$

Since altruistic effort is given by (6): $C^{\prime}\left(a_{2}^{A}\right)=\beta$ and $C^{\prime}(\cdot)$ is increasing function, we have $C^{\prime}\left(a_{2}^{A}\right) \leq \beta$ for all $a \leq a_{2}^{A}$. Taking into account that $C(0)=0$ we have

$$
C\left(a_{2}^{A}\right)=\int_{0}^{a_{2}^{A}} C^{\prime}(a) d a \leq \beta a_{2}^{A} \leq a_{2}^{A}
$$

which guarantees that condition (15)holds. So, the first claim is proved.

For the second claim note that since the Manager makes take-it-or-leave-it offer, the Worker will obtain utility at the outside option level which is utility under non-altruistic relation. So, the first inequality in (13) holds with equality. This establishes the result.

\section{Proof of Lemma 5.}

Proof. If non-altruistic relation is established, the worker gets utility

$$
U_{2}^{N A}=w_{2}^{N A}-C\left(a_{2}^{N A}\right)+\beta v_{2}^{N A}=\overline{\theta_{2 H}^{M}}=\lambda\left(y_{1}-A_{H}\right)+(1-\lambda) \bar{\theta}
$$

since $a_{2}^{N A}=0$ - see (3), $v_{2}^{N A}=0$; and $w_{2}^{N A}=\overline{\theta_{2 H}^{M}}$ because there is competition à-la Bertrand between Principals for establishing the non-altruistic relation.

If the altruistic relation is established, then the wage is set by the Manager according to his belief about the outside option for the worker (see Proposition $4)$ :

$$
(1-\beta) w_{2}^{A}=(1-\beta) \overline{\theta_{2 L}^{M}}-\beta a_{2}^{A}+C\left(a_{2}^{A}\right)
$$

The worker's utility if he accepts the offer is determined by (see (7) )

$U_{2}^{A}=w_{2}^{A}-C\left(a_{2}^{A}\right)+\beta\left(\overline{\theta_{2}^{A}}+a_{2}^{A}-w_{2}^{A}\right)=(1-\beta) w_{2}^{A}-C\left(a_{2}^{A}\right)+\beta\left(\overline{\theta_{2 L}^{A}}+a_{2}^{A}\right)$

which gives after substituting $w_{2}^{A}$ from (17)

$$
U_{2}=(1-\beta) \overline{\theta_{2 L}^{M}}+\beta \overline{\theta_{2}^{A}}=\lambda\left(y_{1}-(1-\beta) A_{L}-\beta a_{1}\right)+(1-\lambda) \bar{\theta}
$$

Comparison of the two utility levels in (16) and (18) gives the result.

\section{Proof of Proposition 1.}

Proof. Consider the game tree - see Figure 2. Let us start from the node marked by $(*)$ where NA relation is established in the 1-st period.

Equilibrium requires that the Altruistic relation is established in the 2-nd period according to Proposition 4 and then effort should be equal to belief: $a_{1}=A_{L}^{N A}$. To support equilibrium, a deviation in the 1-st period effort followed by the choice of NA relation in the 2-nd period should coincide with belief: $a_{1}=A_{H}^{N A}$ at the out of equilibrium path. 


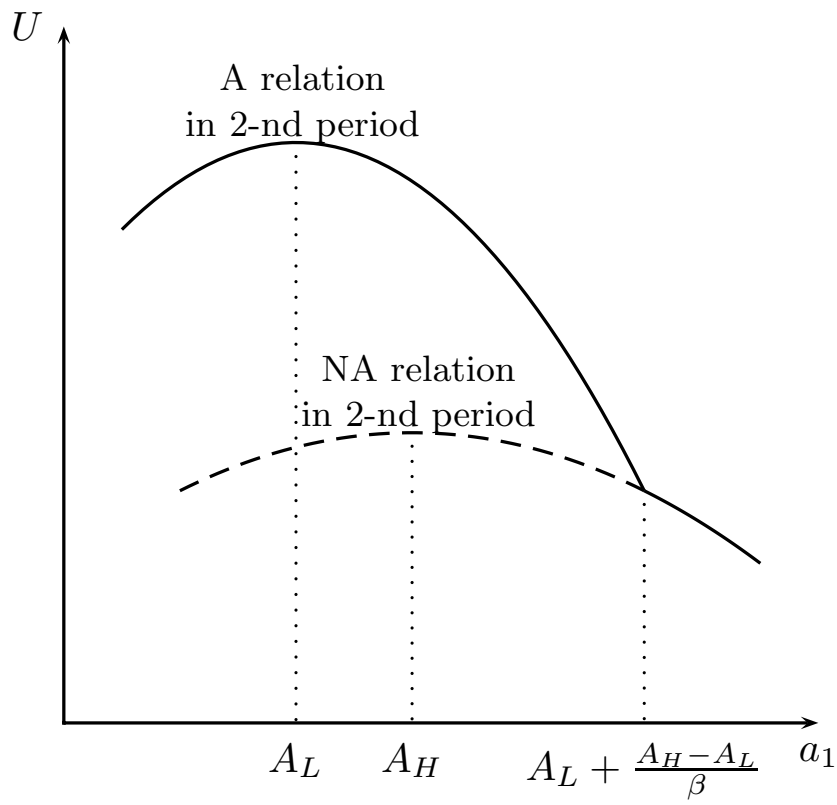

Consider the case $A_{H}^{N A}>A_{L}^{N A}$.

The following picture illustrates the proof.

If the Altruistic relation is going to be established at the 2-nd period, the expected utility of the two periods is ${ }^{3}$ :

$$
U_{A 1}=w_{1}-C\left(a_{1}\right)+\beta\left(\widetilde{y}_{1}-w_{1}\right)+E_{1}^{A}\left[U_{2}^{A}\right]
$$

The term with expectation is computed in Proposition 5:

$$
E_{1}^{A}\left[U_{2}\right]=(1-\beta) \lambda a_{1}+\bar{\theta}-(1-\beta) \lambda A_{L}^{N A}
$$

Substituting this we get

$$
\begin{aligned}
U_{A 1} & =w_{1}-C\left(a_{1}\right)+\beta\left(\widetilde{y}_{1}-w_{1}\right)+(1-\beta) \lambda a_{1}+\bar{\theta}-(1-\beta) \lambda A_{L}^{N A}= \\
& =(1-\beta) \lambda a_{1}-C\left(a_{1}\right)+\left\{\operatorname{const}\left[a_{1}\right]\right\}
\end{aligned}
$$

and the maximization program is

$$
\max _{a_{1} \leq A_{L}^{N A}+\frac{1}{\beta}\left(A_{H}^{N A}-A_{L}^{N A}\right)}\left\{(1-\beta) \lambda a_{1}-C\left(a_{1}\right)+\left\{\text { const }\left[a_{1}\right]\right\}\right\}
$$

Solution should satisfy $a_{1}^{*}=A_{L}^{N A}$ so it has to be internal and is given by

$$
C^{\prime}\left(A_{L}^{N A}\right)=C^{\prime}\left(a_{1}\right)=(1-\beta) \lambda
$$

If NA relation is going to be established in the 2-nd period, then according to Proposition 5

$$
E_{1}^{A}\left[U_{2}\right]=\lambda a_{1}+\bar{\theta}-\lambda A_{H}^{N A}
$$

\footnotetext{
${ }^{3}$ since the non-altruistic relation is established, $\widetilde{y_{1}}$ denotes output obtained by subject-to altruism Principal when another worker works for him. Analogously, the notation $\widetilde{w_{1}}$ is used. Notice that $\widetilde{y_{1}}$ is NOT affected by the altruistic worker effort choice in the period 1
} 
and

$U_{A 1}=w_{1}-C\left(a_{1}\right)+\beta\left(\widetilde{y}_{1}-w_{1}\right)+\lambda a_{1}+\bar{\theta}-\lambda A_{H}^{N A}=\lambda a_{1}-C\left(a_{1}\right)+\left\{\right.$ const $\left.\left[a_{1}\right]\right\}$

The worker maximization program is

$$
\max _{a_{1} \geq A_{H}^{N A}+\left(\frac{1}{\beta}-1\right)\left(A_{H}^{N A}-A_{L}^{N A}\right)}\left\{\lambda a_{1}-C\left(a_{1}\right)+\left\{\text { const }\left[a_{1}\right]\right\}\right\}
$$

Notice that $A_{H}^{N A}>A_{H}^{N A}+\left(\frac{1}{\beta}-1\right)\left(A_{H}^{N A}-A_{L}^{N A}\right)$ since $\beta<1$ and $A_{H}^{N A}>$ $A_{L}^{N A}$. So, $A_{H}^{N A} \notin\left[A_{H}^{N A}+\left(\frac{1}{\beta}-1\right)\left(A_{H}^{N A}-A_{L}^{N A}\right) ;+\infty\right)$. This means that it is never possible to obtain solution $a_{1}^{*}=A_{H}^{N A}$ which means it is never possible to have NA relation established in the 2 -nd period at the equilibrium path.

Now consider the case $A_{H}^{N A} \leq A_{L}^{N A}$. The two maximization programs are the same but now the problem (20) has internal solution

$$
C^{\prime}\left(A_{H}^{N A}\right)=C^{\prime}\left(a_{1}\right)=\lambda
$$

and problem (19) doesn't have solution $a_{1}^{*}=A_{L}^{N A} \in\left[0, A_{L}^{N A}+\frac{1}{\beta}\left(A_{H}^{N A}-A_{L}^{N A}\right)\right]$. Then NA relation is going to be established in the 2-nd period which contradicts Proposition 4. This means that at the equilibrium path the beliefs should satisfy $A_{H}^{N A}>A_{L}^{N A}$.

We have proofed so far that if altruistic relation is going to be established, effort level is $a_{1}=A_{L}^{N A}$. If deviation to $\mathrm{NA}$ relation in the 2 -nd period is considered then utility will not be lower than for effort $a_{1}^{\prime}=A_{H}^{N A}$ because this corresponds to the global maximum of (20).

Compare the 1-st period (maximized) expected utility levels for these two possibilities .

For $a_{1}=A_{L}^{N A}$ and altruistic relation in the 2 -nd period:

$$
\begin{aligned}
U_{A 1} & =w_{1}-C\left(a_{1}\right)+\beta\left(\widetilde{y}_{1}-w_{1}\right)+(1-\beta) \lambda a_{1}+\bar{\theta}-(1-\beta) \lambda a_{1}^{\mu}= \\
& =-C\left(a_{L}^{N A}\right)+w_{1}+\beta\left(\widetilde{y}_{1}-w_{1}\right)+\bar{\theta}
\end{aligned}
$$

For $a_{1}^{\prime}=A_{H}^{N A}$ and NA relation in the 2 -nd period

$$
\begin{aligned}
U_{A 1} & =w_{1}-C\left(a_{1}\right)+\beta\left(\widetilde{y}_{1}-w_{1}\right)+\lambda a_{1}+\bar{\theta}-\lambda A_{H}^{N A}= \\
& =-C\left(A_{H}^{N A}\right)+w_{1}+\beta\left(\widetilde{y}_{1}-w_{1}\right)+\bar{\theta}
\end{aligned}
$$

It is easy to see that the only difference is in the cost of effort. So, the worker prefers (ex-ante) to establish Altruistic relation in the 2-nd period and hence chooses effort $a_{1}=A_{L}^{N A}$ under non-altruistic relation in the 1-st period. Intuition behind this is the following. Extra effort in the 1-st period might lead to extra wage in the 2 -nd period. But since the relation type in the 2 -nd period switches from Altruistic to Non-Altruistic, the extra effort is revealed and wage in the 2-nd period will be adjusted accordingly.

The case of altruistic relation in the 1 -st period (the node ${ }^{* *}$ on the game tree) is considered in the same manner.

This finishes the proof. 


\section{Proof of Proposition 2.}

Proof. 1. To establish the altruistic relation in the 1-st period, the two participation constraints at the 1-st period - for the principal and for the Agent should hold (we omit the index 1 to simplify notation in the proof).

IC for the Worker:

$$
w^{A}-C\left(a^{A}\right)+\beta\left(y^{A}-w^{A}\right)+U_{2}^{A} \geq w^{N A}-C\left(a^{N A}\right)+\beta\left(y^{N A}-w^{N A}\right)+U_{2}^{A}
$$

where the left-hand side is the workers's total (period 1 and 2) utility on the equilibrium path after establishing the altruistic relation in the 1-st period and the right-hand side is the same for the case of non-altruistic relation in the 1-st period.

Taking into account that $y^{A}=\bar{\theta}+A_{L}^{A}, y^{N A}=\bar{\theta}+A_{L}^{N A}, w^{N A}=y^{N A}$, $a^{A}=A_{L}^{A}, a^{N A}=A_{L}^{N A}$

this gives:

$$
w^{A} \geq \bar{\theta}+A_{L}^{N A}+\frac{\Delta C-\beta \Delta a}{1-\beta}
$$

IC for the Principal:

$y^{A}-w^{A}+\alpha\left(w^{A}-C\left(a^{A}\right)\right)+V_{2}^{A} \geq y^{N A}-w^{N A}+\alpha\left(w^{N A}-C\left(a^{N A}\right)\right)+V_{2}^{A}$

this gives:

$$
w^{A} \leq \bar{\theta}+A_{L}^{N A}-\frac{\alpha \Delta C-\Delta a}{1-\alpha}
$$

For the two inequalities (21) and (22) hold simultaneously, the following inequality for the right-hand sides should hold:

$$
\bar{\theta}+a_{L}^{N A}-\frac{\alpha \Delta C-\Delta a}{1-\alpha} \geq \bar{\theta}+a_{L}^{N A}+\frac{\Delta C-\beta \Delta a}{1-\beta}
$$

which gives

$$
\Delta C \leq \Delta a
$$

2. It is clear that

$$
\Delta C=C\left(a_{L}^{A}\right)-C\left(a_{L}^{N A}\right)=\int_{a_{L}^{N A}}^{a_{L}^{A}} C^{\prime}(x) d x=C^{\prime}(\xi) \Delta a
$$

where $\xi$ is some point between $a_{L}^{N A}$ and $a_{L}^{A}$.

Since $C^{\prime}()$ is increasing function, there will be $C^{\prime}\left(a_{L}^{N A}\right)<C^{\prime}(\xi)<C^{\prime}\left(a_{L}^{A}\right)$ where $a_{L}^{N A}<\xi<a_{L}^{A}$

Since $C^{\prime}\left(a_{L}^{N A}\right)=(1-\beta) \delta \lambda$ and $C^{\prime}\left(a_{L}^{N A}\right)=(1-\beta) \delta \lambda+\beta$ one can obtain $C^{\prime}(\xi)=(1-\beta) \delta \lambda+\eta \beta$ where $0 \leq \eta \leq 1$.

Then

$$
\Delta C=C^{\prime}(\xi) \Delta a=((1-\beta) \delta \lambda+\eta \beta) \Delta a
$$

which defines the function $\eta(\beta)$

So, the condition for establishing altruistic relation (23) may be rewritten as

$$
\delta \lambda \leq r(\beta) \equiv \frac{1-\eta(\beta) \beta}{1-\beta}
$$


It is clear from (25) that

$$
\lim _{\beta \rightarrow 0} r(\beta)=1
$$

since $0 \leq \eta \leq 1$ It is also clear that $1-\eta \beta>1-\beta$ unless $\eta=0$ which is the case only if $C^{\prime}$ is constant, so

$$
r(\beta)>1 \text { for } \beta>0
$$

Now let us prove that $r(\beta)$ is increasing function. Consider $\beta_{1}<\beta_{2}$. Let $\Delta C_{j}=C\left(a_{L}^{A}\left(\beta_{j}\right)\right)-C\left(a_{L}^{N A}\left(\beta_{j}\right)\right)$ and $\Delta a_{j}=a_{L}^{A}\left(\beta_{j}\right)-a_{L}^{N A}\left(\beta_{j}\right)$ for given $\delta \lambda>1$ such that altruistic relation is established for $\beta_{1}$, i.e. $\Delta C_{1}<\Delta a_{1}$. First, we prove that $\Delta C_{2}<\Delta a_{2}$.

Notice:

- $C^{\prime}\left(a_{2}^{A}\right)=\beta_{2}+\left(1-\beta_{2}\right) \delta \lambda>1$ since this is weighted average with weights $\beta_{2}$ and $1-\beta_{2}$ of 1 and $\delta \lambda>1$.

- $C^{\prime}\left(a_{1}^{N A}\right)=\left(1-\beta_{1}\right) \delta \lambda<1$. Indeed, if this is not true then $\Delta C=$ $\int_{a_{1}^{N A}}^{a_{1}^{A}} C^{\prime}(x) d x>\Delta a$ because the function $C^{\prime}(x)>1$ for the whole range $x \in\left[a_{L}^{N A}, a_{L}^{A}\right]$ since $C^{\prime}$ is increasing and greater than 1 at the left side of the interval.

- $a_{2}^{N A}<a_{1}^{N A}$ since $C^{\prime}\left(a_{2}^{N A}\right)<C^{\prime}\left(a_{1}^{N A}\right)$ where $C^{\prime}\left(a_{j}^{N A}\right)=\left(1-\beta_{j}\right) \delta \lambda$

- $a_{2}^{A}<a_{1}^{A}$ since $C^{\prime}\left(a_{2}^{A}\right)<C^{\prime}\left(a_{1}^{A}\right)$ where $C^{\prime}\left(a_{j}^{A}\right)=\beta_{j}+\left(1-\beta_{j}\right) \delta \lambda$

Consider now

$$
\begin{gathered}
\Delta C_{2}-\Delta a_{2}=\int_{a_{2}^{N A}}^{a_{2}^{A}} C^{\prime}(x) d x-\int_{a_{2}^{N A}}^{a_{2}^{A}} 1 d x=\int_{a_{2}^{N A}}^{a_{2}^{A}}\left(C^{\prime}(x)-1\right) d x= \\
=\int_{a_{2}^{N A}}^{a_{1}^{N A}}\left(C^{\prime}(x)-1\right) d x+\int_{a_{1}^{N A}}^{a_{1}^{A}}\left(C^{\prime}(x)-1\right) d x-\int_{a_{2}^{A}}^{a_{1}^{A}}\left(C^{\prime}(x)-1\right) d x= \\
=\int_{a_{2}^{N A}}^{a_{1}^{N A}}\left(C^{\prime}(x)-1\right) d x+\left(\Delta C_{1}-\Delta a_{1}\right)-\int_{a_{2}^{A}}^{a_{1}^{A}}\left(C^{\prime}(x)-1\right) d x
\end{gathered}
$$

We have for the first integral $\int_{a_{2}^{N A}}^{a_{1}^{N A}}\left(C^{\prime}(x)-1\right) d x<0$ since $C^{\prime}(x)<1$ for $x \in\left[a_{2}^{N A} ; a_{1}^{N A}\right]$ because $C^{\prime}(x)<C^{\prime}\left(a_{1}^{N A}\right)<1$ for these $x$.

For the last integral we have $\int_{a_{2}^{A}}^{a_{1}^{A}}\left(C^{\prime}(x)-1\right) d x>0$ since $C^{\prime}(x)>1$ for $x \in\left[a_{2}^{A} ; a_{1}^{A}\right]$ because $C^{\prime}(x)>C^{\prime}\left(a_{2}^{A}\right)>1$ for these $x$.

So, finally we obtain

$$
\Delta C_{2}-\Delta a_{2}<\Delta C_{1}-\Delta a_{1}<0
$$


which means $\Delta C_{2}<\Delta a_{2}$.

The function $r(\beta)$ can be considered as the value of $\delta \lambda$ such that $\Delta C=\Delta a$. We have proved that for all $\delta \lambda<r\left(\beta_{1}\right)$ holds $\Delta C_{2}<\Delta a_{2}$. So, to obtain $\Delta C_{2}=$ $\Delta a_{2}$ the value of $\delta \lambda$ should be higher then $r\left(\beta_{1}\right)$, in other words $r\left(\beta_{2}\right)>r\left(\beta_{1}\right)$. So, we obtained that $r(\beta)$ is increasing function.

Finally, consider $\beta \rightarrow 1$. From the definition of $\eta$ in (24) we have $\Delta C=$ $((1-\beta) \delta \lambda+\eta \beta) \Delta a$. It is clear that $\Delta a \rightarrow 1, \Delta C \rightarrow \int_{0}^{1} C^{\prime}(x) d x$, as $\beta \rightarrow 1$. So it's easy to see that

$$
\lim _{\beta \rightarrow 1} \eta(\beta)=\Delta C=C(1)-C(0)
$$

Then

$$
\lim _{\beta \rightarrow 1} r(\beta)=\lim _{\beta \rightarrow 1} \frac{1-\eta \beta}{1-\beta}=\lim _{\beta \rightarrow 1}\left(\eta(\beta)+\frac{1-\eta(\beta)}{1-\beta}\right)=+\infty
$$

This finishes the proof.

\section{References}

Andreoni, James, Philanthropy, in Handbook of the Economics of Giving, Altruism and Reciprocity, Vol. 2, Amsterdam, North Holland, 2006.

Bartling, Bjorn and Ferdinand von Siemens, "The Intensity of Incentives in Firms and Markets: Moral Hazard with Envious Agents," LMU Discussion Papers in Economics, 2006, 14.

Bénabou, Roland and Jean Tirole, "Incentives and Prosocial Behavior," American Economic Review, 2006, 96 (5), 1652-1678.

Bolton, Patrick and Mathias Dewatripont, Contract Theory, MIT Press, 2005.

Dur, Robert and Amihai Glazer, "Optimal Incentive Contracts when Workers envy their Boss," 2004.

Falk, Armin and Michael Kosfeld, "The Hidden Costs of Control," American Economic Review, 2006, 96 (5), 1611-1630.

Fehr, Ernst and Armin Falk, "Psychological foundations of incentives," European Economic Review, 2002, 46 (4-5), 687-724.

- and Bettina Rockenbach, "Detrimental effects of sanctions on human altruism," Nature, 2003, 422, 137-140.

- and Klaus M. Schmidt, Advances in Economics and econometrics, Vol. 1, Cambridge University Press,

Goel, Anand Mohan and Anjan V. Thakor, "Optimal Contracts When Agents Envy Each Other," manuscript, Olin School of Business, 2003.

Grund, Christian and Dirk Sliwka, "Envy and Compassion in Tournaments," Journal of Economics $\mathscr{6}$ Management Strategy, 2005, 14 (1), 187207. 
Holmstrom, Bengt, "Managerial Incentive Problems: A Dynamic Perspective," Review of Economic Studies, 1999, 66 (1), 169-182.

Itoh, Hideshi, "Moral Hazard and Other-Regarding Preferences," The Japanese Economic Review, 2004, 55 (1), 18-45.

Kahneman, Daniel, Jack L. Knetsch, and Richard Thaler, "Fairness as a Constraint on Profit Seeking: Entitlements in the Market," American Economic Review, 1986, 76 (4), 728-741.

Laffont, Jean-Jacques and David Martimort, The Theory of Incentives: The Principal-Agent Model, Princeton University Press, 2002.

Rotemberg, Julio, "Human Relations in the Workplace," The Journal of Political Economy, 1994, 102 (4), 684-717.

_ , "Handbook of the Economics of Giving, Altruism and Reciprocity, volume 2, chapter Altruism, Reciprocity and Cooperation in the Workplace," 2006.

Sliwka, Dirk, "Trust as a Signal of a Social Norm and the Hidden Costs of Incentive Schemes," American Economic Review, 2007, 97 (3), 999-1012. 